\title{
RADIOLOGICAL INVESTIGATION
}

\author{
By JOHN V. SPARKS \\ (Assistant Medical Officer, X-Ray Department, St. Bartholomew's Hospital, E.C.; Radiologist \\ i/c Diagnostic Department, The Royal Cancer Hospital (Free), S.W.3; Assistant Director, \\ Hospital for Consumption and Diseases of the Chest, Brompton, S.W.3.)
}

While it may be true to say that radiology may give the first indication or suspicion of the presence of a neoplasm in the chest, the inference that it is due to carcinoma will frequently be presumptive.

The establishment of an early diagnosis is now of increasing importance, owing to the fact that treatment by pneumonectomy has become practical and recognised as one of the methods of choice.

The diagnosis of carcinoma of the lung is a problem calling for the closest co-operation between the general practitioner, the physician, the radiologist, the laryngologist, the surgeon, and the pathologist, and should not await confirmatory radiological evidence of its spread.

The general practitioner and the physician will be the first to realise that the earlier recognition of malignant disease can only come from a more frequent request for the X-ray examination of the chest, and should invite the help of radiology in patients complaining of a hard, dry, unproductive cough, unexplained dyspnoea or malaise, before the onset of haemoptysis or the finding of abnormal physical signs in the chest.

At the Brompton Hospital a routine X-ray examination of the chest is made on every patient, irrespective of his symptoms, and this routine has now been in force for a number of years, and has frequently been the means of discovering chest disease which would otherwise have remained undetected.

In some cases the lesion has proved to be a carcinoma of the lung, but it can be remarked that this examination, even if negative, can form the most useful piece of evidence should the patient subsequently develop any chest complaint, and though it may not yet be feasible for the general practitioner to have such examinations routinely carried out, one hopes that such facilities will become increasingly practical after the war, for it is in this way that malignant disease of the lung will be recognised at an earlier stage.

It is, indeed, not uncommon to discover such a lesion during the routine examination of the shoulder or abdominal viscera, or during the fluoroscopic examination of the chest which forms an essential part of any barium examination. To-day the introduction of miniature mass radiography will bring under observation a further group of cases for investigation who may be symptomless, or whose symptoms have not yet become sufficiently developed to bring them to consult their doctor. The yearly re-examination by this method will bring such patients under observation at an earlier stage, and will also bring increasingly before the public the value of radiology in the diagnosis of chest disease.

It is a disquieting thought that the most expert auscultation and percussion may fail to reveal a tumour in the lung as large as a tennis ball, even when it is known to be present.

I do not wish to infer that radiology is an infallible means of recognising malignant disease in the chest, and call to mind at once a patient who died from cerebral metastasis where the primary lesion, no larger than a pea, was found at necropsy in the right bronchus, and gave no radiographic indication of its presence: and of another child, aet. 9, with a large abdominal tumour with a small deposit in the periphery of the right lung, thought to be due to a secondary deposit. At the post-mortem the lesion in the lung was found to be the primary growth with an enormous secondary deposit in the right suprarenal gland.

The extreme variation in the initial X-ray appearances of carcinoma of the lung is emphasised when comparing the cases referred to the Brompton Hospital for diagnosis and possible surgical treatment, and those referred to the Royal Cancer Hospital for treatment by radiotherapy.

For descriptive purposes the lesions may be subdivided into those in which the tumour is demonstrated, and those in which the presence of the primary tumour is inferred from secondary phenomena due to its presence. The first group may be subdivided according to whether the tumour is mediastinal or peripheral. 


\section{Peripheral Tumour (Figs. I and II).}

A peripheral tumour of the lung may be seen as a solitary rounded opacity in the lung with clear-cut margins, usually more than an inch in diameter, and must be considered to be malignant until proved to be of a different nature. The visualisation of such a tumour will naturally depend on its size and position, but a lesion of considerable proportions can pass unrecognised if the routine examination fails to include fluoroscopy to locate a neoplasm lurking behind the cardiac shadow, or in that not inconsiderable area of lung tissue obscured by the outline of the domes. A lateral view is essential to localise the position of the tumour, and

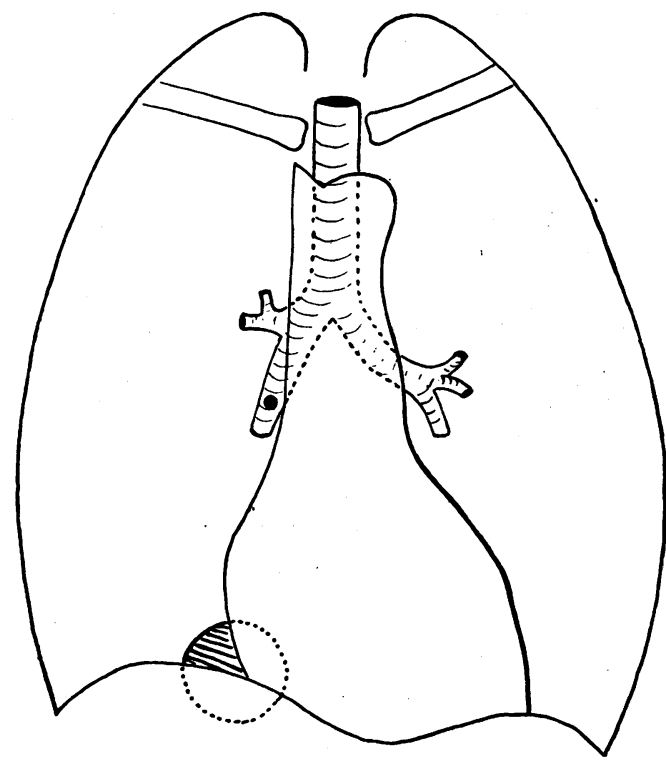

FIG. IA.-Anterior view of peripheral tumour partially obscured by the heart and diaphragm.

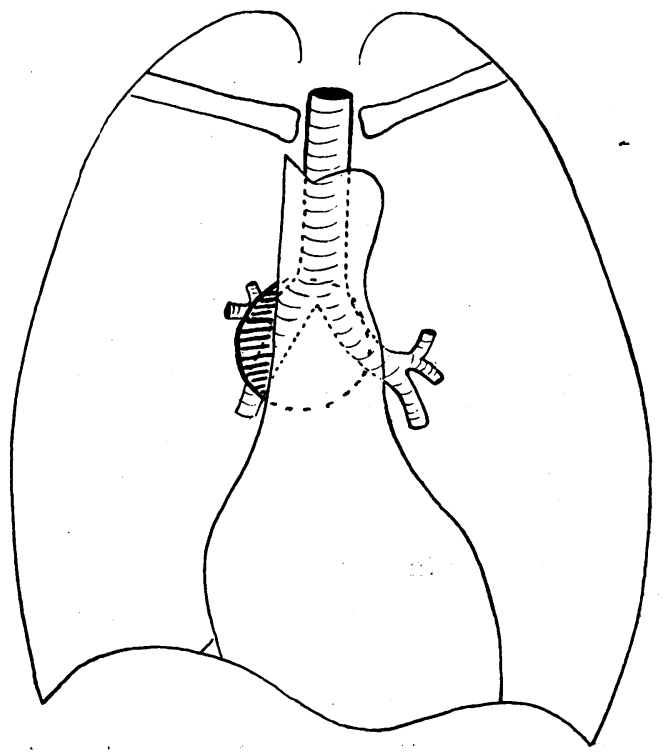

FIG. 2A.-Right " hilar" tumour, see Fig. 2B.

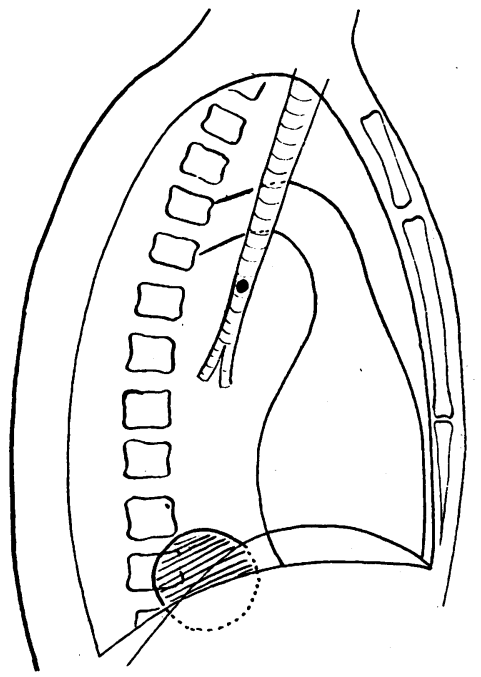

FIG. IB.-Right lateral view to show position of tumour.

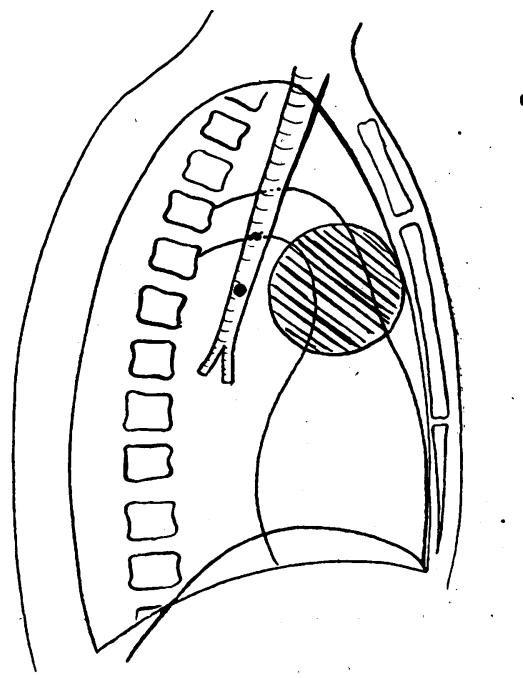

FIG. 2B.-Right lateral view of right " hilar" tumour showing its anterior position. 
sometimes a diagnostic pneumothorax is required to demonstrate that it is intrapulmonary. Such neoplasms tend to spread outwards to involve the ribs by lysis, and radiological evidence of hilar involvement may be indefinite or a late phenomenon.

Differential diagnosis may be difficult as the tumour may be out of reach of the bronchoscope, and must be from an Assman's focus (which may also occur in the lower zone) hydatid cyst, solitary cyst, blood cyst, central pneumonia, encysted interlobar fluid, single secondary deposit, innocent tumour, saccular aneurysm or gumma.

When a peripheral tumour necroses excavation may occur, the appearances simulating very closely those seen in a lung abscess. Tomography is used to demonstrate or exclude the presence of excavation in doubtful cases.

Rounded growths, when seen in the hilar region, are often found to lie well anterior to the hilum of the lung in the upper lobe, and for this reason obstruction of the main bronchi may be late. On reaching the peripheral margin the rounded tumour tends to conform to the shape of the thoracic wall and to lose its uniform convexity in outline. Tumours when more centrally placed may produce lobular areas of atelectasis distal to them.

\section{Pancoast Tumour (Fig. III).}

When the tumour is situated in the apex of the lung its rounded appearance is soon lost, except on its lateral or inferior margin. In this position a tumour has been described by Pancoast as the superior sulcus tumour, and in some cases has been proved to be of bronchogenic origin.

Early involvement of the brachial plexus and branches of the sympathetic nerve (Horner's syndrome) is common, and lateral vertebral erosion and destruction of the upper ribs is seen. In the early stages differentiation must be made from neurofibroma, mediastinal abscess and subclavian aneurysm when the tumour is right sided.

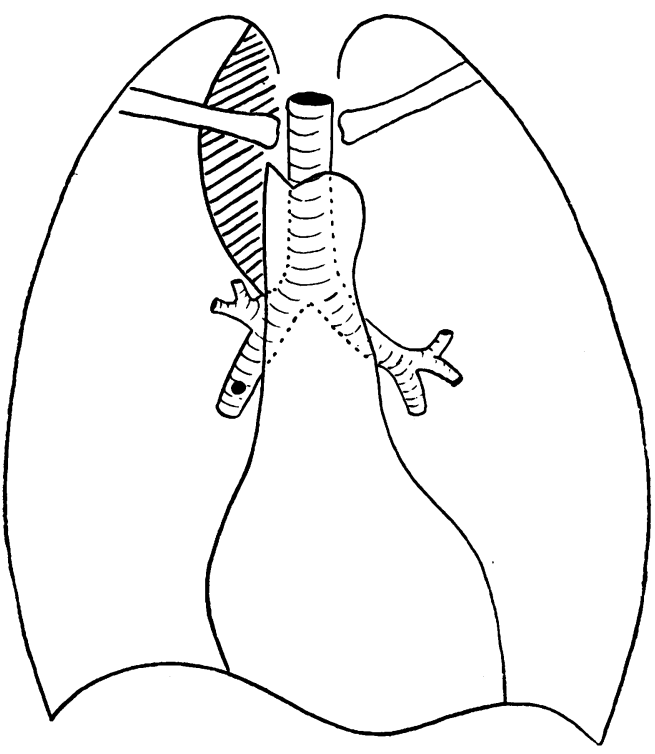

FIG. 3.-Pancoast's tumour.

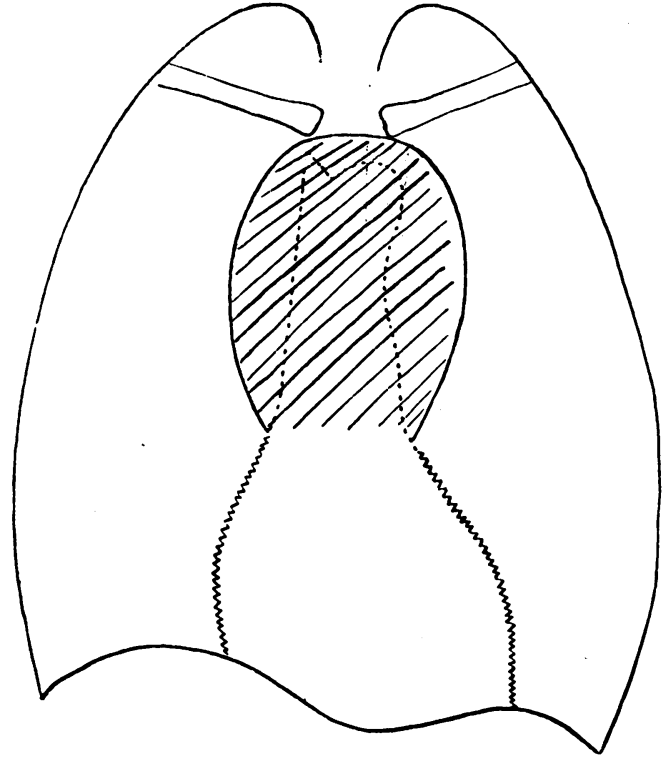

FIG. 4.-Kymographic tracing of mediastinal tumour simulating aneurysm, shown by kymography to be non-pulsatile, and proved by postmortem to be a carcinoma.

\section{Mediastinal Tumour (Fig. IV).}

The second group of cases in which the tumour is visualised are those in which the tumour is situated in the mediastinum, and may reach large proportions and project to the right and left of the midline.

There is often doubt and argument as to whether such tumours arise primarily in the 
mediastinum, or result from massive glandular involvement from a small focus in one of the adjacent main bronchi. In such cases differential diagnosis must be made from aneurysm, mediastinal cyst, e.g. dermoid, lymphadenoma, achalasia, intra-thoracic thyroid, mediastinal effusion, vertebral abscess and tuberculous adenopathy. The mediastinal lymphosarcoma frequently proves on section to be a carcinoma.

Great assistance is obtained in these cases by fluoroscopy and by a barium swallow. Views taken in the oblique and lateral positions are essential, and a penetrating view is often helpful. Emphasis is laid on the fallacy of accepting pulsation on the screen as any proof of aneurysm. Marked pulsation is seen frequently in mediastinal neoplasm, and aneurysms sometimes appear relatively immobile.

The use of the kymograph-a new method which records the pulsations on the film-is now often decisive in distinguishing expansile and transmitted pulsations. The finding of vertebral or sternal erosion due to pressure atrophy is also helpful, and all cases where the Wassermann reaction is negative should be carefully reviewed.

\section{Bronchial Occlusion (Fig. V).}

The commonest group of bronchial carcinomata are those in which the tumour is not visible in the routine radiograph, and whose presence is revealed as the result of the changes which are produced in the surrounding lung tissues. Such growths arise commonly in the major bronchial divisions and very rarely in the trachea itself. The first suspicion of their presence may be aroused sometimes by a unilateral hilar enlargement, sometimes a phrenic paralysis, sometimes an unexplained cardiac displacement towards the affected side, or sometimes an area of localised emphysema due to ball valve action. Any of these signs may form a sufficient indication for an early bronchoscopy, especially in cases where a previous routine radiograph demonstrates that they are of recent occurrence. Succeeding comparative views may demonstrate a lobular or lobar area of abnormal opacities resulting from incomplete or unsustained bronchial occlusion.

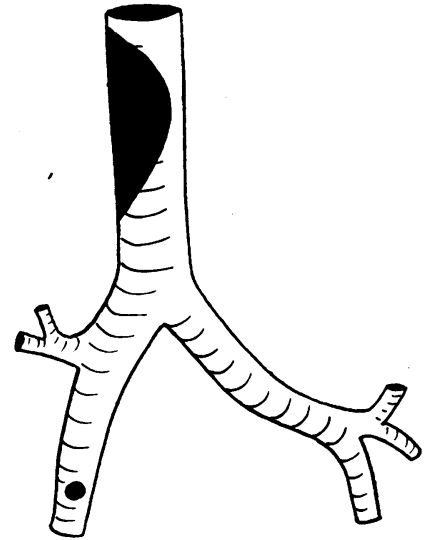

FIG. 5.-Diagrammatic representation of the tomograph in the case of Mrs. M., aged 70, who had suffered from haemoptysis and choking sensation. Bronchoscopy by Mr. Mill revealed a fungating growth at this site. Six months after radio-therapy at the Royal Cancer Hospital tomograph and bronchoscopy showed that the growth had disappeared.

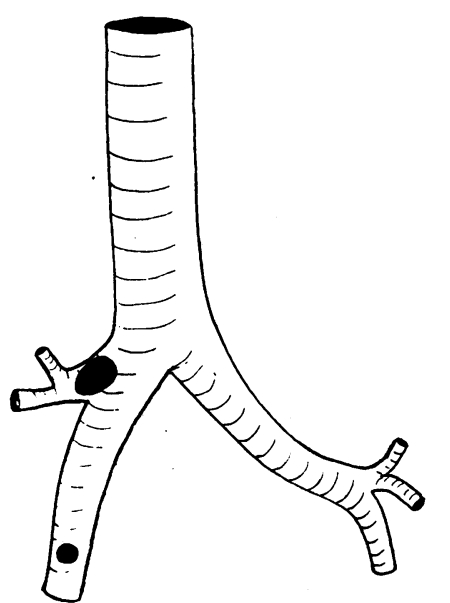

FIG. 6.-Diagrammatic representation of tomograph of bronchial adenoma in a patient under the care of Dr. Geoffrey Todd at the Midhurst Sanatorium, and now removed with the right upper lobe by Mr. A. Tudor Edwards.

\section{Bronchography.}

At this stage bronchography is an important aid to demonstrate the presence and site of the bronchial block. One side only should be filled, and the patient carefully manipulated so that the oil has every chance of filling the suspected area. Radiographs are taken in the supine, erect and lateral positions, and the block should be confirmed in each of the three views. 


\section{Bronchoscopy (Fig. VI).}

The result of this examination may form a useful guide to the bronchoscopist, but whether the bronchographic findings are positive or negative, a bronchoscopy will still be required to demonstrate the nature of the obstruction and to obtain a biopsy.

Tomography has, on occasions, brought into view a small tumour in one of the main bronchial divisions, which are clearly outlined by this means. Differential diagnosis is not,

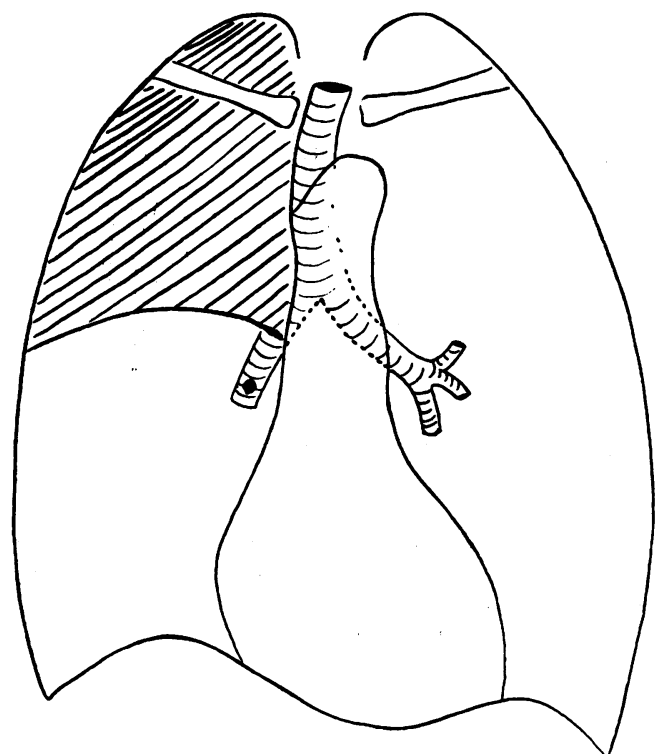

FIG. 7A.-Atelectasis of the right upper lobe following an obstruction of the right eparterial bronchus.

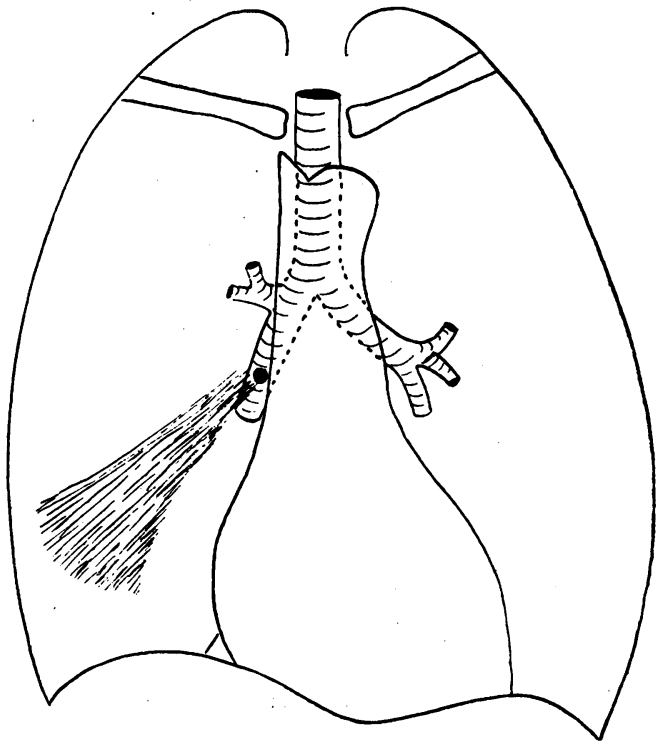

FIG. 8A.-Atelectasis of the right middle lobe.

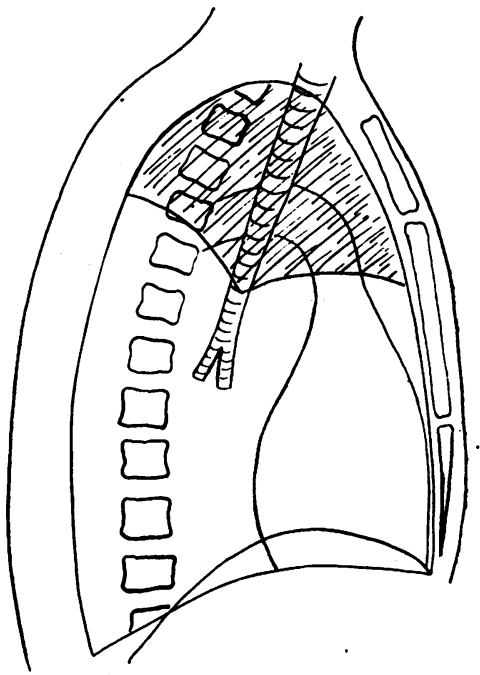

FIG. 7B.-Lateral view of same.

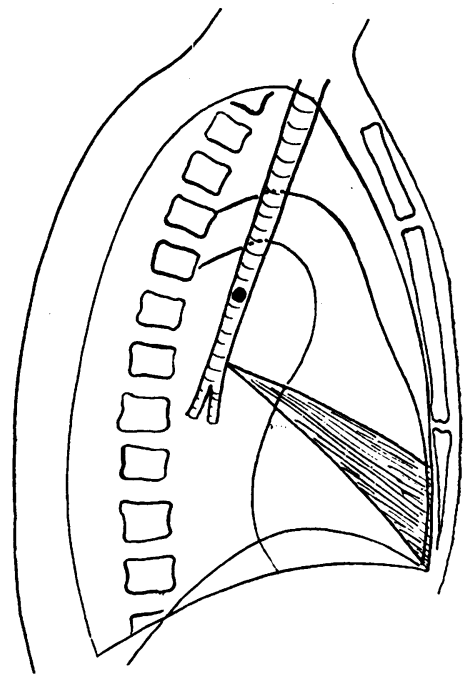

FIG. 8B.-Lateral view of same. 


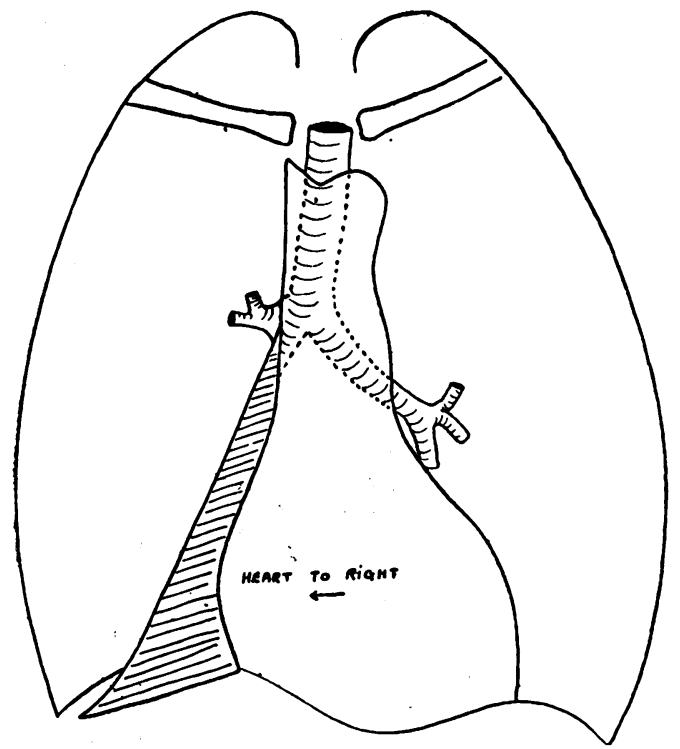

FIG. 9A.-Atelectasis following obstruction to the right lower lobe bronchus below the origin of the middle lobe bronchus.

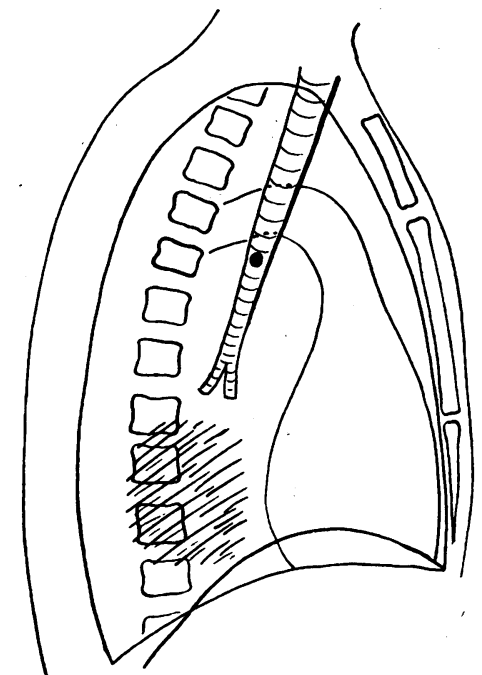

Fig. 9B.-Lateral view of same. Note lack of lobar distribution.

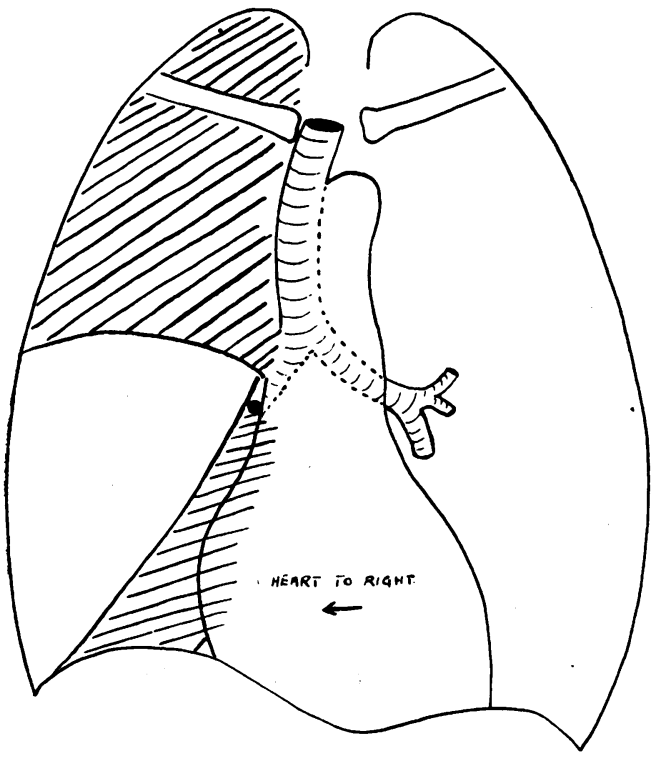

FIg. IOA.-Appearances seen following atelectasis of the right upper and lower lobes.

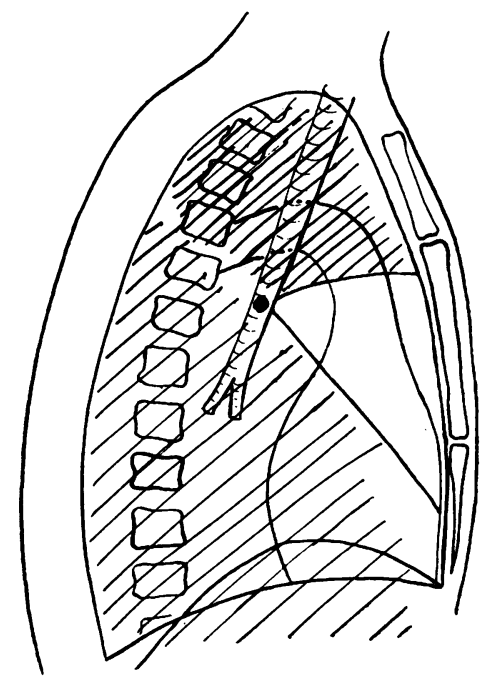

FIG. IOB.--Lateral view of same. 


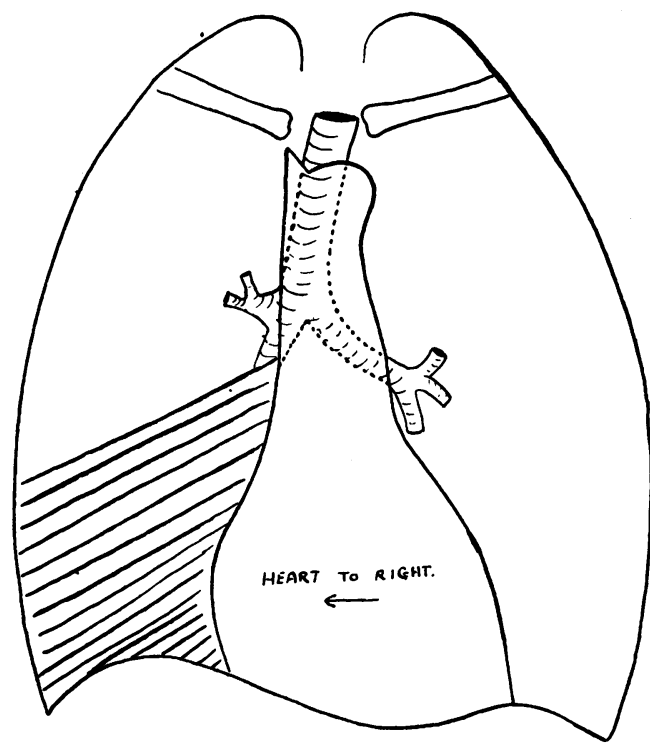

FIG. IIA.-Appearances seen following atelectasis involving the right middle and lower lobes.

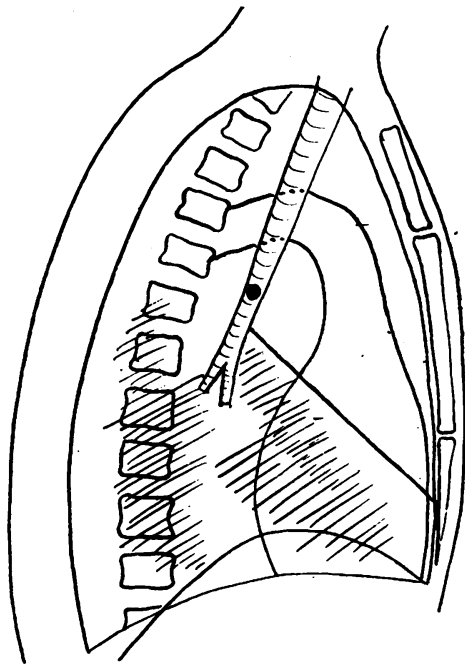

FIG. I B.-Lateral view of same.

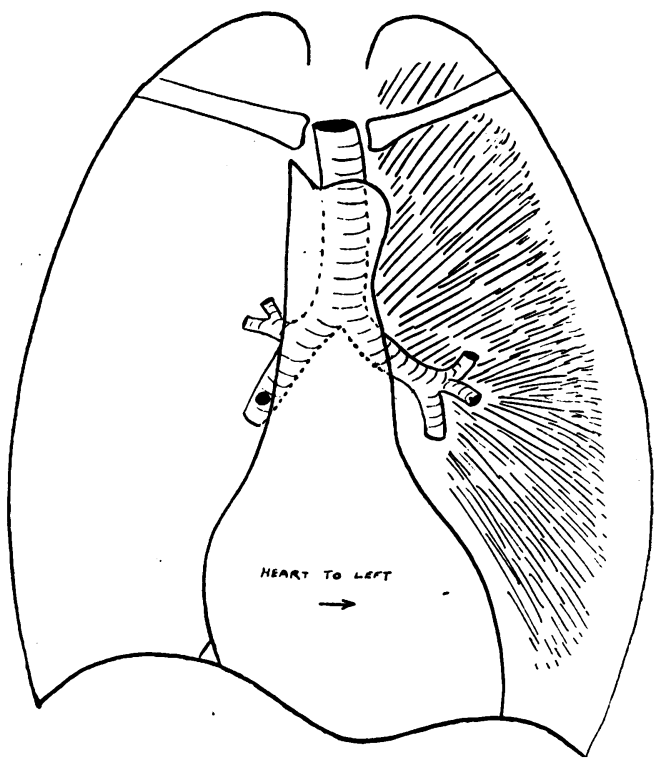

FIG. I2A.-Appearances seen following obstruction to the left upper main bronchus.

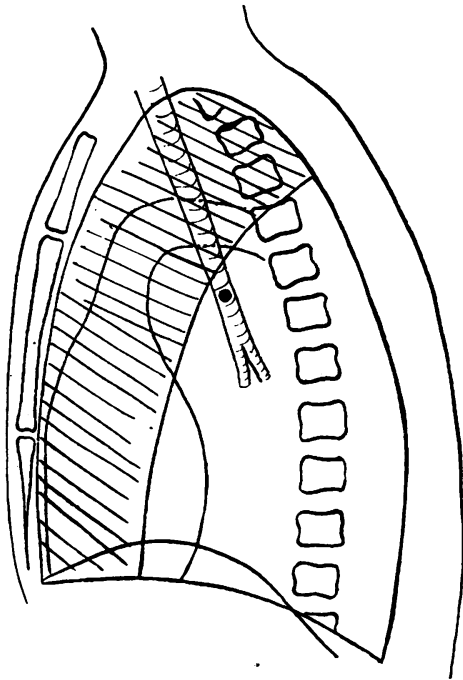

FIG. I2B.-Lateral view of same. 
as indicated, radiological, and it is of the greatest importance to realise the need for early bronchoscopy. The same initial X-ray appearances may be seen in phthisis, resolving pneumonia or bronchiectasis, which complications may, in fact, be present.

Many cases do not present themselves for X-ray examination until atelectasis is complete (Figs. 7-13). This is commonly lobar, or may be lobular, or may involve adjacent portions of two lobes, depending on the site of the primary tumour and the bronchi involved by its unrelenting spread. Mediastinal involvement or pleural effusion may occur early or late, and phrenic paralysis is frequently associated. When lobar, the margins of the lobe are at first demarcated, but not always sharply, and the degree of contraction of the upper lobe is usually minimal as compared with that usually seen in the middle or lower lobes, which contract down to a fraction of their usual size. The displacement of the heart and mediastinum towards the affected side may be slight in the upper lobe block, and appreciable when the lower lobe is involved.

At this stage the differential diagnosis is again made by bronchoscopy, and this examination may be aided by tomography and bronchography to demonstrate the site of the obstruction.

The same initial X-ray appearances can be seen in foreign body inhalation, occlusion by an inflammatory gland, inflammatory ulceration in a bronchus, as in tubercle, stricture of a bronchus, innocent neoplasm, obstruction by a mucus plug with resulting bronchiectasis, or in lobar pneumonia. The finding of bronchiectasis is common, and frequently accompanies neoplasm. Progress is unfortunately not long delayed, and within a few weeks or months the lesion will usually spread to obstruct the main bronchi of the remaining lobes on the affected side. This may come from direct spread of the main growth, or as a result of pressure or invasion from its glandular metastases. Sometimes the right middle lobe is the last lobe to come deflated by reason of the anterior origin of its bronchus.

When the atelectasis involves the whole lung the heart and mediastinum are usually displaced to the affected side, but may regain a normal position in the presence of an effusion: the opacity will be relatively homogeneous, but becoming less dense peripherally owing to the diminished thickness to be penetrated. If the block has been present for some weeks clear spaces may be seen within it indicating bronchial dilatation.

Differential diagnosis when first seen at this stage will be from innocent tumour, foreign body, aneurysm causing complete occlusion (usually left-sided), pneumonic consolidation, empyema and fibrosis with bronchiectasis.

Bronchography has on more than one occasion revealed flattening and widening of the left main bronchus where aneurysm was responsible for the obstruction.

Empyema is important, as in the absence of classical symptoms its presence may have been clinically overlooked. Mediastinal displacement towards the affected side may be misleading, but the ribs will often show periosteal thickening.

The bronchogram will show compression of the lung without bronchial occlusion.

I hope that I have indicated the important role which radiology plays in the demonstration of and subsequent investigation of the abnormal

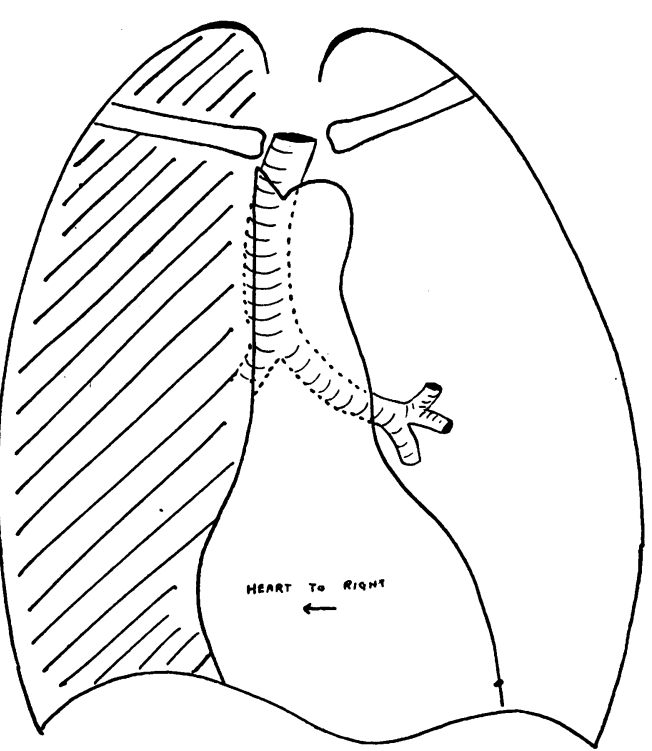

FIG. 13.-Appearances seen following obstruction to the right upper and lower bronchus. shadows which may be suggestive of neoplasm in the chest, and the need for close co-operation to establish an early diagnosis.

NoTE.-In the diagrams which have been kindly prepared for me by N.M.D., no attempt has been made to reproduce the appearances due to pleural involvement, rib destruction, lobular atelectasis, excavation or contiguous lobar extension of malignant disease, or of hilar or mediastinal deposits. 